\title{
Lingüistica digital: revisión de su estado actual y retos en el Instituto Universitario de Investigación en Lingüistica Apli- cada de la Universidad de Cádiz
}

\author{
MARIo CRESPo Miguel \\ Departamento de Filología/Instituto Universitario \\ de Investigación en Lingüística Aplicada \\ Universidad de Cádiz \\ Avda. Dr. Gómez Ulla, nº 1 \\ 11003 Cádiz \\ E-mail: mario.crespo@uca.es
}

LINGÜÍSTICA DIGITAL: REVISIÓN DE SU ESTADO ACTUAL Y RETOS EN EL INSTITUTO UNIVERSITARIO DE INVESTIGACIÓN EN LINGÜÍSTICA APLICADA DE LA UNIVERSIDAD DE CÁDIZ

RESUMEN: Este trabajo presenta el papel que está adquiriendo la lingüistica digital en el Instituto Universitario de Investigación en Lingüística Aplicada de la Universidad de Cádiz. Esta disciplina tiene el objetivo de complementar la forma de trabajar tradicional con nuevos instrumentos, enfoques, procedimientos de análisis, y modo de acceder y almacenar la información. Para ello se analiza su concepción, los diferentes tipos de estudio que engloba y su influencia en las labores de investigación, formación y la prestación de servicios de este instituto. A la luz de los datos que se ofrecen en este artículo, se pude comprobar cómo este centro de investigación tiene un papel paradigmático en estos nuevos derroteros de la lingüística en el siglo XXI.

PALABRAS CLAVES: lingüistica digital; tecnologia; lingüistica aplicada; instituto de investigación; recursos tecnológicos.

SUMARIO: 1. Introducción. 2. La lingüistica digital y recursos tecnológicos. 3. El Instituto Universitario de Investigación en Lingüística Aplicada. 3.1. Proyectos de investigación. 3.2. Formación e innovación docente. 3.3. Equipamientos y servicios de transferencia del Instituto. 4 Lineas futuras. 5. Conclusiones.
DIGITAL LINGUISTICS: A REVIEW OF ITS STATUS AND CHALLENGES AT THE UNIVERSITY INSTITUTE OF RESEARCH IN APPLIED LINGUISTICS OF THE UNIVERSITY OF CADIZ

ABSTRACT: This paper presents the role that Digital Linguistics is playing in the University Institute of Research in Applied Linguistics of the University of Cadiz. This trend in Linguistics aims to complement the traditional way of researching with new tools, approaches, analysis procedures, and way of accessing and storing information. In this work, we try to define it, analyse the different types of studies that belong to it and how it is influencing the way researching, teaching and transferring services in this institute. This paper shows how this research centre can be considered exemplary in the new paths of Linguistics in the $21^{\text {st }}$ century

KEY WORDS: digital linguistics; technology; applied linguistics; research institute; technology resources.

SUMMARY: 1 . Introduction. 2. Digital Linguistics and Technology resources. 3. University Institute of Research in Applied Linguistics. 3.1. Research projects. 3.2. Training and teaching innovation. 3.3. Equipment and transfer services. 4. Future lines. 5. Conclusions.
LINGUISTIQUE NUMÉRIQUE : RÉVISION DE SON STATUT ET DE SES DÉFIS A L'INSTITUT UNIVERSITAIRE DE RECHERCHE EN LINGUISTIQUE APPLIQUÉE DE L'UNIVERSITÉ DE CADIX

RÉSUMÉ : Ce document présente le rôle que la linguistique numérique est en train d'acquérir à l'Institut Universitaire de Recherche en Linguistique Appliquée de l'Universite de Cadix. Cette tendance vise à compléter la manière traditionnelle de travailler avec de nouveaux outils, approches, procédures d'analyse et moyens d'accès et de stockage de l'information. Pour celà, sa conception de la linguistique numérique est analysée, ainsi que les différents types d'études qu'elle englobe et son influence sur la recherche, la formation et la prestation de services de cet institut. Suite aux données proposées dans cet article, il est possible de voir comment ce centre de recherche a un rôle paradigmatique dans ces nouvelles orientations de la linguistique au $21^{\text {ème }}$ siècle.

MOTS CLÉS : linguistique numérique ; technologie ; linguistique appliquée ; institut de recherche ; ressources technologiques.

SOMMAIRE : 1 . Introduction. La lin guistique numérique et les ressources technologiques. 3. l'Institu de Recherche en Linguistique Appliquée. 3.1 Projets de recherche. 3.2 Formation des enseignants et innovation. 3.3. Equipement et services de transfert de l'Institut. 4. Lignes futures. 5. Conclusions.
Fecha de Recepción Fecha de Revisión Fecha de Aceptación Fecha de Publicación
$09 / 10 / 2020$

$23 / 10 / 2020$

$01 / 11 / 2020$

$01 / 12 / 2020$ 


\title{
Lingüistica digital: revisión de su estado actual y retos en el Instituto Universitario de Investigación en Lingüistica Aplicada de la Universidad de Cádiz
}

\author{
MARIO CRESPO MigUeL
}

\section{INTRODUCCIÓN}

La tecnología está adquiriendo un papel fundamental en todos los campos del saber. Los recursos tecnológicos son las herramientas que permiten el empleo útil y provechoso del conocimiento. Ante esta realidad, la lingüística ha comenzado a interesarse por las posibilidades que ofrece lo digital. Las tecnologías y herramientas del lenguaje nos hacen ser más precisos en las observaciones, hacen posible analizar aspectos no observables anteriormente, proveen de nuevas utilidades a esta sociedad de la información y, en muchos casos, permiten además mejorar la calidad de vida de las personas. Hoy es ya casi imposible pensar en lingüística sin pensar en las industrias de la lengua, fonética, lexicografia, lingüística de corpus, psicolingüística, neurolingüística, enseñanza de lengua, lingüística forense, terminología o traducción, por poner algunos ejemplos. la sinergia entre lingüística y tecnología está aún en sus albores y prevemos un futuro lleno de vías para la investigación y el desarrollo.

El monográfico Lingüística y los recursos tecnológicos tiene la intención recoger parte de las comunicaciones presentadas en las XVI Jornadas de Lingüística, celebradas los días 24 y 25 de octubre de 2018 y organizadas por los miembros del Ârea de Lingüística General de la Universidad de Cádiz. Las Jornadas de Lingüística, cuya andadura se inició en 1995, han pretendido siempre ofrecer un foro de debate, discusión e intercambio científico de ideas entre profesores y alumnos sobre los aspectos más relevantes y desarrollos más recientes de la Lingüística moderna, con una exposición y puesta al día de las principales lineas y proyectos de investigación que actualmente se están llevando a cabo. Igualmente, estas sesiones siempre han estado destinadas al fomento de las relaciones con numerosos lingüistas españoles y extranjeros, así como, desde una perspectiva docente, a acercar a los alumnos, postgraduados, doctorandos y universitarios en general, a los temas básicos de la actualidad lingüística. Esta labor ha supuesto la organización ya de dieciséis eventos académicos, y la posterior publicación de sus resultados (destacamos las publicadas desde 2010: Casas Gómez y García Antuña, 2012; Casas Gómez y Sánchez-Saus Laserna, 2013; Casas Gómez y Vela Sánchez, 2017; Casas Gómez, Díaz Hormigo y Crespo Miguel, 2018).

Cuando hacemos referencia al Área de Lingüistica General de la Universidad de Cádiz es indiscutible asociarla con el grupo interuniversitario y consolidado del Plan Andaluz de Investigación denominado Semaínein ${ }^{1}$, fun-

\footnotetext{
1 https://hum147.uca.es/.
} 
dado en 1994, y con su director, el Prof. Dr. Miguel Casas Gómez. Su prestigio nacional e internacional viene avalado por la puesta en marcha de relevantes proyectos de investigación, así como por numerosas publicaciones valoradas muy positivamente en los distintos procesos de evaluación externa de la productividad científica, en los que ha obtenido la máxima puntuación en todas sus convocatorias del Plan Andaluz de Investigación, Desarrollo e Innovación ${ }^{2}$. Además, las investigaciones del grupo sobresalen especialmente por sus excelentes resultados en la transferencia dentro del campo de las Humanidades. De su labor se deriva, del lado docente, la creación de unos estudios de Licenciatura de Lingüística, su reconversión posteriormente en Grado $^{3}$ y la implantación de un programa de Doctorado ${ }^{4}$ sobre la misma materia. Asimismo, debemos destacar la consecución de numerosos proyectos de investigación, una larguísima lista de publicaciones científicas, la potenciación de la revista Pragmalingüística, la organización de multitud de jornadas, coloquios y congresos, la apuesta por la transferencia de los resultados de la investigación a través de convenios y contratos I+D con empresas e instituciones, y la creación del Instituto de Lingüística Aplicada ${ }^{5}$ (Varo Varo, 2018).

En esta monografia se recogen diferentes trabajos que exponen diferentes ámbitos de aplicación, posibles líneas de investigación y visión sobre la tecnología en su confluencia con la Lingüística. Sus autores proceden de diferentes universidades españolas: la Profa. Dra. Chelo Vargas Sierra de la Universidad de Alicante nos presenta "La estación de trabajo del traductor en la era de la inteligencia artificial. Hacia la traducción asistida por conocimiento"; el Prof. Dr. Javier González Rosa de la Universidad de Cádiz presta atención a "Aplicaciones de la neurofisiología cognitiva y la estimulación cerebral no invasiva al estudio del lenguaje"; la Profa. Dra. Sheila Queralt Estévez del Laboratorio SQ-Lingüistas Forenses se ocupa de "El uso de recursos tecnológicos en Lingüística Forense", el Prof. Dr. Fernando Trujillo Sáez de la Universidad de Granada expone "Tecnología para la Enseñanza y el Aprendizaje de Lenguas Extranjeras. La Enseñanza de lenguas Asistida por Ordenador. Pasado, presente y futuro", y concluye la Profa. Dra. María Luisa Carrió Pastor de la Universitat Politècnica de València con "Conocer la lengua a través de corpus: METOOL, retos para el análisis de los marcadores discursivos".

En estas páginas de introducción al monográfico, queremos dar a conocer el papel que está adquiriendo el Instituto Universitario de Investigación en Lingüística Aplicada de la Universidad de Cádiz dentro de lo que se conoce actualmente como lingüística digital. La siguiente sección analiza su concepción y los instrumentos que aporta para el análisis lingüístico, para centrarnos posteriormente en la labor que realiza este instituto a nivel nacional

\footnotetext{
2 https://www.paidi2020.es/.

${ }^{3}$ https:// filosofia.uca.es/grado-en-linguistica-y-lenguas-aplicadas/.

${ }^{4} \mathrm{https}$ ///escueladoctoral.uca.es/doctorado/oferta-doctorado/programa-de-doctorado-enlinguistica-8208/.

${ }^{5}$ https://ila.uca.es.
} 
e internacional. Como veremos, la lingüística digital no es más que el resultado natural de la progresiva incorporación de los avances informáticos en la lingüística. Se trata de una tendencia al alza, como augura el crecimiento de los trabajos relacionados con esta temática.

Finalmente, queremos dar las gracias en esta introducción a los compañeros del Área de Lingüística General de la Universidad de Cádiz que se ofrecieron para presentar y moderar las sesiones de las XVI Jornadas de Lingüística, así como a todas aquellas otras personas, organismos y entidades que, con su colaboración y ayuda, hicieron posible que se pudiera realizar este ciclo de conferencias: Vicerrectorado de Investigación (actualmente Vicerrectorado de Política Científica y Tecnológica), Departamento de Filología, Decanato de la Facultad de Filosofia y Letras, Instituto Universitario de Investigación en Lingüística Aplicada y Grupo Semainein del Plan Andaluz de Investigación.

\section{LA LINGÜISTICA DIGITAL Y RECURSOS TECNOLÓGICOS}

Cada vez está más claro el papel esencial que desempeña la informática en el desarrollo de las ciencias humanas (Auger, Drouin y L'Homme, 1991). Desde su surgimiento en la década de los cuarenta, el uso de ordenadores empezó lentamente a incorporarse a todo tipo de tareas científicas, entre ellas la investigación lingüística. Desde mediados de los 80, con la universalización de los ordenadores, surgen nuevos métodos de análisis, lo que conlleva un incremento cuantitativo y cualitativo de los estudios lingüísticos que incorporan estos instrumentos. El salto al estudio asistido por computadora permite a los investigadores procesar muchos más datos que los métodos tradicionales (Nerbonne y Tonelli, 2017). Desde sus orígenes, la rama informática de la lingüística no solo ha estado interesada en la creación de modelos computacionales para su incorporación a aplicaciones prácticas (Crystal, 2008), sino también en ser soporte para el estudio científico del lenguaje (Richards y Schmidt, 2010), y en ser capaz de procesar automáticamente las lenguas (Johnson y Johnson, 1998).

Cuando hablamos de lingüistica digital debemos entender un conjunto de técnicas y herramientas enfocadas a la manipulación de datos lingüísticos en formato digital, lo que incluye el almacenamiento, la representación, la manipulación y la difusión de este tipo de datos. También se ocupa de cómo extraer y representar la información lingüística en forma electrónico, así como de las mejores prácticas para trabajar con esos datos, utilizando todos los beneficios de las tecnologias actuales. La lingüística digital no es sinónimo de lingüística computacional, aunque ciertas habilidades y métodos de investigación pueden superponerse entre estas dos disciplinas. Mientras que la lingüística computacional se ocupa principalmente de la modelización y el procesamiento del lenguaje en aplicaciones informáticas, el campo emergente de la lingüística digital es más amplio, al proporcionar un conjunto completo de fundamentos científicos, metodológicos y prácticos para el estudio del lenguaje en la era digital. 
De esta manera, los actuales avances en el área de la computación están multiplicando exponencialmente los recursos disponibles para el análisis lingüístico, filológico o literario (Berry, 2011; Boto Bravo, 2017). Cada día aparecen nuevas herramientas digitales y programas de análisis disponibles, lo que ofrece oportunidades para la investigación (Vogel, Hamann y Gauer, 2018). La ventaja decisiva de estos métodos asistidos por computadora es el control de la intuición mediante la adopción de técnicas empíricas de observación. Aunque la intuición de los hablantes nativos es una presunción irremplazable para las suposiciones cualitativas sobre el uso de la lengua, esta puede fallar, por lo que hay casos en los que no es adecuada. Los algoritmos y programas informáticos desarrollados van a ser un soporte de las diferentes ramas del saber lingüístico y pueden ser usados para discernir patrones del lenguaje en sus diferentes niveles. Cuando analizamos las herramientas de las que dispone la lingüística digital, podemos agruparlas en tres grandes bloques:

1. Programación orientada al procesamiento de texto. Se trata de la forma básica para el procesamiento del lenguaje natural. Tiene la ventaja de que permite la creación "a medida" de herramientas para el análisis de los diferentes niveles lingüisticos. Entre los lenguajes de programación destaca el lenguaje $\mathrm{R}$, que ha adquirido un apoyo considerable dentro de las Humanidades Digitales (Arnold et al., 2019). Este lenguaje está últimamente muy presente en talleres y cursos especializados de investigación. Una de sus mayores ventajas es su gran desarrollo estadístico y la disponibilidad de múltiples paquetes para tratar todos estos tipos de datos en un solo entorno. Otro lenguaje de programación de amplio uso en lingüistica es Python. Este lenguaje de programación es orientado a objetos e interpretado. Su principal fortaleza reside en su facilidad, y en poseer un conjunto potente y maduro de librerías para todo tipo de tareas. Python dispone de una amplisima comunidad de usuarios (Madnani, 2007), al ser uno de los lenguajes más aprendidos a nivel mundial (Ozgur et al., 2017).

2. Herramientas de visualización. Este tipo de métodos informáticos facilita el análisis macroscópico de los fenómenos lingüisticos en un corpus de estudio. Permite visualizar mediante gráficos, patrones y tendencias de estilo y contenido vastas franjas de datos textuales. Como algunos investigadores observan, es "como tener un telescopio que te hace ver galaxias completamente nuevas" (Varsava, 2018). Asimismo, el Text Analysis Portal for Research (Tapor) ${ }^{6}$ (Rockwell et al., 2020), un recurso accesible para la búsqueda de herramientas de análisis textual, lista actualmente unas 500 herramientas diferentes para este fin, desglosadas

\footnotetext{
${ }^{6}$ http://tapor.ca/. Fecha de acceso: mayo de 2020.
} 
por facilidad de uso, tipo de licencia (gratuita, comercial, Creative Commons, etc.) o tipo de análisis (visualización, anotación o procesamiento básico).

3. Herramientas de análisis experimental. Para Hemforth (2017), este tipo de lingüística se caracteriza por abordar los fenómenos lingüísticos con métodos experimentales, es decir, consiste en el estudio lingüístico basado en pruebas cuantitativas. Esta evidencia puede ser experimental en su sentido estricto o derivada de corpus de texto. En cualquier caso, esta perspectiva entiende que la validez de las hipótesis debe probarse utilizando estadísticas inferenciales para poder sacar conclusiones generales a partir de una muestra aleatoria de participantes o expresiones lingüísticas, o ambas cosas. Tradicionalmente el enfoque experimental ha sido más o menos constante en fonética y psicolingüística, más recientemente está demostrando ser útil en la morfología, la sintaxis, la semántica, pragmática y en toda la lingüística aplicada. Entre sus herramientas encontramos análisis espectográficos, electroencefalogramas, ecógrafos, técnicas de eyetracking, plataformas terminológicas, estaciones de traducción, interfaces de exploración de corpus o técnicas de digitalización de documentos en alta resolución.

Nos hallamos por tanto ante un nuevo paradigma en la investigación sobre el lenguaje y las lenguas. La lingüística actual se encuentra ante el reto de asumir todos estos nuevos medios e ir integrándolos en su quehacer investigador. Los siguientes apartados desarrollan la posición del Instituto de Investigación en Lingüística Aplicada de la Universidad de Cádiz ante este desafio y desglosan la progresiva integración de este nuevo enfoque digital en sus proyectos, la formación y en los equipamientos con los que cuenta.

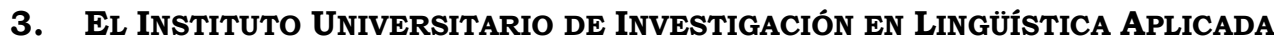

El Instituto Universitario de Investigación en Lingüística Aplicada de la Universidad de Cádiz (ILA) es un centro de investigación, formación, transferencia y prestación de servicios relacionados con el lenguaje ${ }^{7}$. Este centro fue aprobado en Consejo de Gobierno el 17 de diciembre de 2012, certificado por la Junta de Andalucía el 30 de junio de 2014 (como primer Instituto oficial de Andalucia), tras obtener la más alta calificación de excelencia en el informe final de evaluación para su creación, y constituido oficialmente el 19 de noviembre de 2014. Forma parte de un área de especialización estratégica pionera para la UCA (Varo Varo y Casas Gómez, 2018) debido a que se relaciona con la existencia del único grado en Andalucía en Lingüística y Lenguas Aplicadas (y una de las tres, junto con la de Barcelona y la Complutense de Madrid, que ofrece estos cursos en todo el país), y a que es el

\footnotetext{
7 https://ila.uca.es.
} 
segundo existente en España, junto con el IULA ${ }^{8}$, por lo que el Instituto es distintivo y específico en la comunidad andaluza. La conformación técnicocientifica del Instituto se organiza en torno a cuatro gabinetes especializados y dos laboratorios, con autonomía propia pero interdisciplinares:

1) Gabinete de asesoría lingüística.

2) Gabinete de lingüistica clínica.

3) Gabinete de lingüística forense.

4) Gabinete de industrias de la lengua.

5) Laboratorio de fonética.

6) Laboratorio de lingüística computacional y digital.

Entre los objetivos de todos estos gabinetes y laboratorios se encuentra la investigación en todos los ámbitos de la lingüística, la formación de investigadores y técnicos especializados, la organización de cursos, talleres y seminarios de formación y perfeccionamiento, la colaboración con empresas e instituciones a través de los diversos servicios técnicos, y ser un foro de referencia científico nacional e internacional. Como se puede observar en su organización, la lingüística digital se encuentra entre las prioridades de este centro. La finalidad principal del Laboratorio de lingüística computacional y digital es el estudio empírico del lenguaje, la búsqueda y aplicación de tecnologías de la información y la comunicación a la lingüística, y el desarrollo de nuevas herramientas digitales para el análisis y procesamiento del lenguaje y las lenguas. Sus líneas de investigación son:

- Diseño, análisis y procesamiento de corpus lingüísticos y desarrollo de aplicaciones. Esto incluye el trabajo con grandes bancos de datos lingüísticos (textos, grabaciones, diccionarios, etc.), así como instrumentos de análisis y explotación de estos datos (segmentadores, etiquetadores, analizadores sintácticos, etc.).

- Investigación en el ámbito de la lingüística computacional, en cuanto campo multidisciplinar de la lingüística que utiliza la informática para estudiar y tratar el lenguaje humano.

- Estudio de las bases cientificas, de las técnicas y de las herramientas necesarias para un estudio holístico de la comunicación digital.

A pesar de que muchos de los miembros del Instituto provienen de una investigación de corte metodológico tradicional en su origen, podemos observar en general una progresiva incorporación de estos nuevos métodos e instrumentos en su quehacer investigador. Así, esta nueva tendencia aparece de manera transversal en todo el ILA, como muestran sus proyectos, resultados de investigación, transferencia de conocimientos y equipamientos. Los siguientes apartados dan cuenta de cómo es esta simbiosis entre lo lingüístico y lo digital, hasta el punto de que el ILA puede ser considerado

\footnotetext{
8 https://www.upf.edu/es/web/iula.
} 
paradigma de estos nuevos derroteros de la lingüística en el siglo XXI. Para ello, se exponen sus diferentes proyectos de investigación, formación, equipamientos y servicios de transferencia en los últimos años y los previstos en el futuro.

\subsection{PRoyectos De investigación}

En primer lugar, presentamos los proyectos de investigación realizados o en proceso de ejecución dentro del Instituto de Lingüística Aplicada. Del total de proyectos, solo se han seleccionado aquellos que tenían una impronta tecnológica, de acuerdo con las líneas establecidas en los epígrafes anteriores de este trabajo. Estos proyectos abarcan desde instrumentos para la recopilación, almacenamiento, recuperación y difusión de información, hasta entornos de trabajo adaptados a las necesidades de los lingüistas o el uso de herramientas de visualización de datos.

Entre los proyectos más importantes, destacamos, en primer lugar, "Comunicación especializada y terminografia: usos terminológicos relacionados con los contenidos y perspectivas actuales de la semántica léxica (FFI201454609-P)", del Ministerio de Economía y Competitividad, con plazo de ejecución del 1 de enero de 2015 al 31 de diciembre de 2018, y una dotación de 54.540 euros. Este proyecto tiene como finalidad la elaboración de un léxico de los usos terminológicos mediante su integración en una estación terminológica al modo de base de datos relacional. Este sistema unifica corpus de estudio, ontología de términos y visualización de estos en su contexto (Casas Gómez, 2018; Ramírez Salado y Álvarez Torres, 2018). La tecnología mejora así el trabajo de campo del especialista.

Desde el lado de la explotación bibliométrica y de textos, encontramos el proyecto "Evaluación y monitorización de la Comunicación para el Desarrollo y el Cambio Social en España: diseño de indicadores para la medición de su impacto social (CSO2014-52005-R), perteneciente al Programa Estatal de Generación del Conocimiento del Ministerio de Economía y Competitividad y con una subvención de 19.740 euros para el trienio 2015-2018. Este proyecto tiene el objetivo de evaluar el campo de la comunicación para el desarrollo y el cambio social en España con el fin de reforzar su incidencia social (Marí Sáez, 2016). Tomando como punto de partida una doble estrategia metodológica (análisis bibliométrico y estudio de casos), el proyecto hace uso de técnicas estadísticas y de lingüística de corpus para analizar la presencia de este tipo de publicaciones en la investigación sobre comunicación en España (Ceballos Castro, Sánchez-Saus Laserna y Marí Sáez, 2018).

El desarrollo de entornos multilingües y multimodales mediante el uso de las TIC han sido objetivo del proyecto "Weinapp: Sistema multilingüe de información y recursos vitivinícolas (FFI2016-79785-R)", del Ministerio de Economía, Industria y Competitividad durante los años 2016, 2017, 2018 y 2019 con una dotación de 30.250 euros. WeinApp es un proyecto de inves- 
tigación interdisciplinar sobre terminología vitivinícola, cuyo objetivo fundamental es la elaboración de un sistema de información sobre el dominio de la vitivinicultura (Balbuena Torezano, 2018).

En una línea parecida se halla "VIP: Sistema integrado Voz-texto para IntérPretes" del Ministerio de Economía y Competitividad, financiado con 66.550 euros para el periodo comprendido entre el 20 de diciembre de 2017 y el 30 de diciembre de 2020. Este proyecto quiere transformar el sector de la interpretación, mediante la creación de un entorno de trabajo para intérpretes que tenga un impacto similar al que han tenido las tecnologias lingüísticas en traducción. Para ello, se pretende analizar el grado de automatización necesario que requieren estos profesionales, asi como inventariar y analizar las tecnologías existentes de interpretación asistida (Corpas Pastor, 2017).

Finalmente, el proyecto "Sociedade Informacional: memória e tecnologias", financiado por CAPES PRINT (Brasil) con 25.000.000,00 BRL (5.889.697,74 euros) para el periodo del 1 de enero de 2019 al 1 de julio de 2022, engloba a un consorcio internacional (con integrantes de los siguientes países: Brasil, Alemania, Austria, Dinamarca, España, Francia, Holanda, Italia, Portugal, Reino Unido y Suecia, Argentina, Chile, Colombia, Cuba, Estados Unidos y México) con la finalidad de estudiar cuestiones relacionadas con la memoria en el contexto de la Sociedad de la Información, y las diferentes tecnologias utilizadas para recopilarla, almacenarla, recuperarla y difundirla.

\subsection{FORMACIÓN E INNOVACIÓN DOCENTE}

Los nuevos medios tecnológicos están provocando, de forma ya irreversible, un nuevo orden económico y social que condiciona el modo de ser y estar en el mundo, y al que dificilmente podrán las personas mantenerse ajenas (García, Ruiz y Domínguez, 2007). En este entorno económico, social y cultural nace, crece y se educa una generación en pleno desarrollo influida por la presencia de las TIC (Ferreiro, 2006). La universidad y el ILA no son ajenas a estos cambios en la sociedad, por lo que integran el ámbito digital desde diferentes perspectivas en la formación del alumnado y del personal investigador. De este modo, desde el curso 2013-14 funciona el doctorado en lingüística. Se trata de un programa con mención de calidad y con un itinerario formativo vinculado en sus líneas de actuación tanto al ILA como a la Red Nacional de Lingüística. Este doctorado está coordinado por el Área de Lingüistica General de la UCA, y está conformado, además, por relevantes investigadores de las universidades de Valencia, Santiago de Compostela, Complutense y Autónoma de Madrid, así como por reconocidos especialistas de universidades europeas, como Lovaina y Heidelberg. No existen otros estudios de doctorado en lingüística en la comunidad autónoma andaluza. Cuenta con cinco líneas y equipos de investigación, entre las que destaca: 
"Comunicación y aplicaciones tecnológicas de la Lingüística"9. Esta rama se centra en el diseño de estrategias lingüísticas y de asesoramiento para lograr la adecuación comunicativa, oral y escrita, en diferentes ámbitos de la comunicación, así como en el tratamiento automático del lenguaje y la formalización del mismo, de acuerdo con las nuevas tecnologías y las actuales propuestas de la lingüística computacional.

Entre los cursos de formación permanente del ILA, queremos destacar los relativos al convenio de colaboración con el Instituto Superior de Estudios Lingüísticos y Traducción (ISTRAD) y su Máster en Traducción Audiovisual: localización, subtitulación y lenguaje (modalidad virtual y modalidad semipresencial). En este título se imparte el seminario de "Traducción automática" y el módulo de formación permanente en "Traducción y nuevas tecnologías". Otros cursos puntuales de formación en el seno del Instituto han tratado temas como la "Iniciación a las nuevas tecnologias para la investigación en lingüística clínica" o la "Introducción a la lingüística de corpus".

Desde la óptica de la innovación docente, son ya numerosos los proyectos ejecutados desde el ILA. En ellos la tecnología funciona bien como soporte para la docencia y el contacto con los alumnos, como por el empleo de nuevos medios de comunicación y formatos para la enseñanza. Estos proyectos han sido financiados por la Unidad de Innovación Docente de la Universidad de Cádiz y se pueden dividir por su finalidad educativa:

1. Los enfocados a la creación de materiales digitales. Aquí destacan "Diseño de materiales docentes de libre acceso a través de RODIN y OCW para las asignaturas del Área de Lingüistica General de la Universidad de Cádiz" para el curso 2014-2015 con el objetivo de elaborar materiales docentes en formato digital para su divulgación y la mejora de la competencia en lingüística del alumnado; "Píldoras formativas para la mejora del trabajo de fin de grado en el Grado de Lingüística y Lenguas Aplicadas" para el curso 2017-2018, donde se plantea la creación de tutoriales audiovisuales de corta duración (5 min) como un recurso formativo para los jóvenes estudiantes, ampliamente familiarizados con este tipo de formato similar al utilizado en YouTube (Crespo Miguel y Sánchez-Saus Laserna, 2020), o "Recursos digitales para la docencia de la asignatura 'Lingüistica" para el curso 2018-2019, donde se trata de explotar la plataforma educativa de la Universidad de Cádiz, denominada Campus Virtual, mediante la exploración de todo su potencial en las clases presenciales de la asignatura de "Lingüística" de primer curso de la Facultad de Filosofia y Letras de la Universidad de Cádiz que aglutina unos 250 alumnos.

\footnotetext{
${ }^{9}$ https: //escueladoctoral.uca.es/doctorado/oferta-doctorado/programa-de-doctorado-en-linguistica-8208/lineas-de-investigacion-programa-de-doctorado-en-linguistica-8208/.
} 
2. Proyectos para la mejora de la comunicación entre profesores y alumnos. Entre ellos destaca por importancia "Redes sociales para el fomento de la participación, el trabajo autónomo y la motivación de los alumnos", que se ha ido desarrollando durante los cursos 2015-2016, 2016-2017, 2017-2018, 2018-2019. Este proyecto enfatiza el gran potencial que tienen las redes sociales para favorecer el diálogo y el intercambio de conocimientos e ideas entre alumno y profesor, y entre los propios alumnos. Las redes sociales son un medio idóneo para la comunicación y la difusión de contenidos que no tienen cabida durante las horas de clase y, así, aumentar el interés y la motivación del alumno hacia los contenidos propios del grado. Este proyecto ha recibido mención de reconocimiento en algunos de sus cursos (Sánchez-Saus Laserna y Crespo Miguel, 2018; Sánchez-Saus Laserna, 2019). Igualmente, encontramos en este segundo bloque de actuaciones en innovación docente a "Botseriu, chatbot para la resolución de dudas de clase" para los cursos 2019-2020 y 2020-2021. Este proyecto pretende aprovechar las posibilidades comunicativas e informativas de los chatbots para aplicarlo en un contexto docente real de la Universidad de Cádiz. Su principal misión consiste en resolver dudas frecuentes, ayudar en el estudio de la asignatura y convertirse en una fuente de motivación para los alumnos primer curso (Crespo Miguel, 2019).

\section{Proyectos para el desarrollo de competencias digitales en los alum-} nos. En esta línea se encuentra el proyecto "Actividades de creación audiovisual en la asignatura 'Lingüística' de los grados filológicos", realizado durante el curso 2018-2019. El empleo de redes sociales se ha convertido en los últimos años en una actividad cotidiana para la gran mayoría de jóvenes y, por ende, para los universitarios. La propuesta de este proyecto de innovación es que el alumno haga sus trabajos expositivos en el aula usando estos nuevos medios de difusión social.

\subsection{EQUIPAMIENTOS Y SERVICIOS DE TRANSFERENCIA DEL INSTITUTO}

Ese apartado expone los equipamientos del Instituto de Investigación en Lingüística Aplicada y los servicios de transferencia que se derivan de ellos. Desde sus orígenes, el ILA ha ido dotándose con los equipos y programas informáticos necesarios para su funcionamiento y adecuación en la era digital. Su consecución supone una apuesta decidida por el uso de estas nuevas herramientas en la investigación en lingüística. En este sentido, podemos destacar el proyecto "Equipamiento Científico-Técnico del Instituto Universitario de Investigación en Lingüística Aplicada” (UNCA13-1E-2058), del Subprograma Estatal de "Infraestructuras Científicas y Técnicas y Equipamiento" (convocatoria 2013) del Ministerio de Economía y Competitividad, financiado con la ayuda de 95.494,14 euros y plazo de ejecución del 1 de enero de 2013 al 31 de diciembre de 2015. Con este proyecto, se dotó al Gabinete de lingüística forense y al Laboratorio de lingüística computacional 
y digital con una sala multimedia compuesta por 40 puestos de ordenador, diferentes corpus anotados con información lingüística, accesorios informáticos y el software especializado BATVOX para lingüística forense, la herramienta más avanzada de biometría de voz. De esta primera dotación se deriva el Servicio de Peritaje Lingüístico del Gabinete de lingüística forense del ILA, el cual se orienta al análisis de textos orales y escritos en un procedimiento judicial. Entre sus tareas se halla la interpretación y/o desambiguación de cláusulas, la detección de plagio, la atribución de autoría, la identificación de locutor, la transcripción de grabaciones o la autentificación de grabaciones.

En línea con el proyecto anterior, encontramos "Adecuación a las necesidades de infraestructura, gestión informática y comunicación digital de los gabinetes del Instituto Universitario de Investigación en Lingüistica Aplicada", del Programa de Fortalecimiento de la I+D+i de la Universidad de Cádiz (convocatoria 2014) de la Secretaría General de Universidades, Investigación y Tecnología de la Junta de Andalucía. Tuvo una financiación de $36.281,12$ euros y un plazo de ejecución del 6 de noviembre de 2014 al 31 de diciembre de 2015. Con la subvención de este segundo proyecto se adquirió el escáner cenital Zeutschel OS 12002 Advanced Plus, adscrito al Gabinete de asesoria lingüística. La implantación de este escáner ha hecho posible desarrollar el servicio de digitalización de alta resolución de documentos y la incorporación para su manejo de un técnico de apoyo a la investigación (PTA) en la convocatoria del MINECO.

Otro proyecto de especial relevancia en lingüística experimental es "Herramientas y recursos para la formación especializada en el Gabinete de lingüística clínica del ILA", de la Convocatoria Equipa 2016 de los Vicerrectorados de Infraestructuras y Patrimonio y Recursos Docentes y de la Comunicación de la Universidad de Cádiz y una financiación de 3.542,43 euros. Se trata de un proyecto que colabora con la Unidad de Neurociencia y Rehabilitación del Hospital Puerta del Mar de Cádiz. Su ejecución permitió la compra del equipo de electroencefalografia de Bionics, modelo Brainamps de 32 canales, y el desarrollo del servicio de electroencefalografia para el registro de señales EEG y potenciales evocados de respuesta electrofisiológica ante la presentación de estímulos visuales y auditivos.

Además de conseguir estas ayudas, que han permitido dotarse con la tecnología necesaria para el desarrollo de diferentes proyectos y servicios, el ILA viene participando en la convocatoria anual de la Noche Europea de los Investigadores, un proyecto de divulgación científica promovido por la Comisión Europea dentro de las acciones Marie Sklodowska-Curie del programa Horizonte 2020. El objetivo de este evento es acercar la ciencia y la labor de los investigadores a la sociedad en general. Igualmente interviene en el "Foro Europeo para la Ciencia, Tecnología e Innovación (Transfiere)", dedicado al campo de la transferencia y resultados de la investigación en el ámbito social y empresarial. Se trata este del contexto idóneo para dar a conocer los servicios ofrecidos por el ILA a otras universidades, grupos de 
investigación y empresas sobre temas relacionados con la ciencia, la tecnología y la innovación. Finalmente, relacionado también con el ámbito de las empresas, el Instituto fue galardonado con los premios atrÉBT, en la modalidad: "atrÉBT! Human" de los años 2018 y 2019 con el proyecto empresarial CAST, que implementaba una prueba interactiva oral de inglés por ordenador para evaluar el nivel oral de los candidatos, según el Marco Común Europeo de Referencia para las Lenguas (MCERL) y sin necesidad de tener un examinador presente, y el proyecto empresarial C3BOT, una spin-off de creación de chatbots (robots capaces de simular una conversación) para la promoción y difusión de empresas. Este premio está organizado por el Vicerrectorado de Política Científica y Tecnológica de la Universidad de Cádiz y está destinado a fomentar el espíritu emprendedor, apoyar la creación de nuevas empresas y aumentar el impacto socioeconómico de las actividades de investigación y transferencia de conocimiento.

\section{LÍNEAS FUTURAS}

Un acercamiento a los planes futuros de este centro permite entrever un reforzamiento de los proyectos, la formación, los equipamientos y los servicios de transferencia existentes. De esta manera, el proyecto "Lingüística y Humanidades Digitales: base de datos relacional de documentación lingüística (Ref. PY18-FR-2511)" financiado por la Junta de Andalucia con 71.800 euros para el trienio 2020-2022, posee un claro carácter interdisciplinar dentro del campo de las nuevas tecnologías de la información y la representación documental. Su objetivo principal es la digitalización, catalogación y clasificación temática de un conjunto de materiales bibliográficos de carácter lingüístico para la conformación de un relevante repositorio digital que estará disponible en la futura biblioteca del Instituto Universitario de Investigación en Lingüística Aplicada. Este proyecto se relaciona con la organización de la sección XV del XXIII Deutscher Hispanistentag10,"Nuevos ambientes de la hispanística: Digitalización, Reinscripciones, Interfaces", organizado por Universidad de Graz y que cuenta con una sección dedicada a la "Terminología y la lingüística digital". El objetivo de este congreso es mostrar las nuevas formas y herramientas de investigación y enseñanza hispanas en entornos digitales. Entre ellas se hallan la digitalización, el procesamiento, la evaluación digital o el uso de interfaces.

En el ámbito de la lingüística experimental destaca "Language as a neurocognitive evaluation tool for the child population affected by low-prevalence chromosomopathies: a longitudinal study (Chromoling)", del Programa Estatal de I+D+i Orientada a los Retos de la Sociedad (MINECO) con 78.807 euros para el periodo comprendido entre el 1 de enero de 2020 y el 31 de diciembre de 2022. Entre sus integrantes se encuentra el Hospital Punta de Europa de Algeciras y tiene como objetivo el estudio del procesamiento lin-

\footnotetext{
10 https://hispanistentag-2021.uni-graz.at/es/secciones/.
} 
güístico en niños afectados por cromosomopatias de baja prevalencia mediante técnicas psicolingüisticas (test normalizados, pruebas de electroencefalografia y registro ocular), de evaluación neuroanatómica y de genética. Con esto se quiere conocer con más exactitud sus debilidades y fortalezas a nivel cognitivo y lingüístico, y consecuentemente, diseñar herramientas de rehabilitación que faciliten a los afectados la adquisición de las destrezas lingüísticas que les son más problemáticas.

En el apartado de equipamientos, el Instituto contará con una Unidad de Lingüística Experimental. Esta nueva sección se financia a partir del proyecto "Equipamiento científico-técnico de la unidad de lingüística experimental del instituto de lingüística aplicada", dotado de Laboratorio de fonética, Sala de electroencefalograma y Sala de eyetracking (EQC2018-004760P), del Subprograma Estatal de Infraestructuras de Investigación y Equipamiento Científico-Técnico (Plan Estatal I+D+I 2017-2020) con una dotación de 278.913,83 euros. El objetivo de la Unidad de Lingüistica Experimental es la investigación lingüística desde las perspectivas de la producción articulatoria y del procesamiento acústico-perceptivo de los fenómenos del lenguaje. Se busca un acercamiento experimental integral al funcionamiento de las lenguas. De este equipamiento, se espera una potenciación de la investigación y desarrollo de servicios adaptados a los nuevos derroteros de la lingüística.

Finalmente, en el apartado de formación, el Instituto reforzará las líneas docentes existentes y analiza la posibilidad de desarrollar el "Máster Universitario en Lingüística Aplicada”, siguiendo su Plan Director. Se trata de la salida natural para capacitar a las nuevas generaciones en investigación, conocimiento en infraestructuras y transferencia. Esto permitirá a los alumnos profundizar en los problemas más importantes de la lingüística aplicada en su relación con la sociedad: Educación, aplicaciones digitales, Medicina, ámbito jurídico, plurilingüismo, medios de comunicación, comunicación multimodal, tecnología, etc.

\section{Conclusiones}

Asistimos desde hace ya años a una progresiva tecnologización de todas las áreas de conocimiento, y pocos discuten ya que este hecho esté cambiando fundamentalmente la forma en que nos involucramos en el proceso de investigación. En el ámbito del lenguaje y las lenguas, estos nuevos instrumentos tienen el objetivo de complementar la forma de trabajar tradicional con nuevos enfoques, procedimientos de análisis y modo de acceder y almacenar la información. Las universidades están llamadas a la integración de estas nuevas herramientas, por lo que el Instituto de Investigación en Lingüística Aplicada de la Universidad de Cádiz puede considerarse un ejemplo de la integración de estas nuevas tecnologias en el ámbito investigador, docente y de servicios.

Desde el punto de vista de los proyectos y la investigación, las nuevas tecnologias se encuentran entre los campos prioritarios de los Programas 
Marco de la Unión Europea. Encontramos una decidida apuesta en el ILA por la integración de estas nuevas tendencias en el marco de las letras. Todos los proyectos mencionados se caracterizan por una concepción de la tecnología en sentido amplio. Además, como explica Navarro Tomás et al. (2016) y como se puede apreciar en la configuración de los proyectos que hemos presentado, esta forma de trabajar va a suponer hablar de interdisciplinariedad, colaboración y nuevas prácticas aplicadas a las disciplinas humanísticas tradicionales, de la que surge una nueva forma de interpretar y representar la realidad, que complementa a las ya existentes. Los proyectos referidos están formados por equipos de investigadores pertenecientes a diferentes disciplinas, lo que posibilita el aprovechamiento del objeto de estudio desde diferentes perspectivas científicas. De la sinergia entre diferentes campos del saber surgen objetos más complejos y una explicación de la realidad con mayor profundidad.

Respecto al ámbito docente, el ILA contempla en sus prioridades la formación de expertos en lingüística mediante la creación de cursos, talleres y seminarios. En el ámbito de la innovación docente, hemos podido ver cómo las nuevas tecnologias no solo permiten la ampliación de la clase a otros contextos fuera del aula (González-Martínez, Fortuño \& Espuny-Vidal, 2016) sino que facilitan incluir nuevos contenidos que tendrian dificil cabida en un desarrollo tradicional de una asignatura (Poza-Luján et al. 2014). Además, el ámbito lingüístico se ha visto favorecido por el hecho de que muchos de estos nuevos instrumentos estén orientados a la comunicación, por lo que son fácilmente integrables en la metodología de enseñanza del aula de lengua y de idiomas.

Estos proyectos de innovación son imprescindibles para una correcta integración y exploración de todas sus posibilidades. Igualmente, consideramos necesario el desarrollo de líneas de especialización tipo máster o doctorado, que puedan formar a los profesionales del futuro. Se trata del medio esencial para la formación de los nuevos lingüistas. Finalmente, la sociedad demanda que el mundo universitario sea capaz de transferir sus conocimientos a la sociedad y al mundo empresarial. Es por ello que el ILA hace un decidido esfuerzo por colaborar con empresas privadas e instituciones públicas, así como en dar a conocer sus resultados de la investigación en foros y encuentros. De la implementación de equipamientos y su puesta en conocimiento, no solo se mejoran los resultados en investigación, sino que se generan servicios a la sociedad.

Queremos concluir con la reflexión de Presner (2009) quien indica que la tecnologia debe aprovechar los principales puntos fuertes metodológicos de las Humanidades: atención a la complejidad, y capacidad de profundidad analitica, crítica e interpretación. Los algoritmos no pueden entender las estructuras semánticas de los datos que analizan, por lo que no pueden suplantar el razonamiento cualitativo. No se trata de hacer que la lingüistica sea digital, sino de hacer lingüística asistida por lo digital, un campo de actuación para el tratamiento, visualización y almacenamiento de datos lingüísticos de cualquier naturaleza. De esta manera, la lingüística digital está 
modificando sustancialmente la manera de acercarse al hecho lingüístico y probablemente esté ayudando a la aparición de un nuevo paradigma científico en esta área.

\section{REFERENCIAS}

ARNOLD, T., BALLIER, N., LISSÓN, P., \& TILTON, L. (2019): "Beyond lexical frequencies: using $\mathrm{R}$ for text analysis in the digital humanities", Language Resources and Evaluation, 53(4), pp. 707-733.

AUGER, P., DROUIN, P., \& L'HOMME, M.C. (1991): "Automatisation des procédures de travail en terminographie", Translators' Journal, 36(1), pp. 121-127.

Balbuena Torezano, M. C. (2018): “Terminología y traducción en contextos especializados (alemán-español): el sector vitivinícola", EPiC Series in Language and Linguistics, 3, pp. 65-71.

BERRY, D. (2011): "The computational turn: Thinking about the digital humanities", Culture machine, 12.

BOTO BRAVO, M. A. (2017): "Mapa estilométrico de la narrativa de Eduardo Mendoza: aproximación a un análisis estilístico computacional de textos literarios", Epos: Revista de filología, (33), pp. 99114.

JOHNSON, K., \& JOHNSON, H. (eds.) (1998): Encyclopedic dictionary of applied linguistics, Oxford: Blackwell Publishing.

CASAS GÓMEZ, M., \& GARCÍA ANTUÑA, M. (2012): XII Jornadas de Lingüística: Cádiz, del 30 de marzo al 1 de abril de 2009, Cádiz: Servicio de Publicaciones de la Universidad de Cádiz.

CASAS GÓMEZ, M. \& SÁNCHEZ-SAUS LASERNA, M. (2013): XIII Jornadas de Lingüística: Cádiz 15, 16 y 17 de marzo 2010, Cádiz: Servicio de Publicaciones de la Universidad de Cádiz.

CASAS GÓMEZ, M. \& VELA SÁNCHEZ, R. (2017): Eugenio Coseriu, in me- moriam. XIV Jornadas de Lingüística (1st ed.), Cádiz: Servicio de Publicaciones de la Universidad de Cádiz.

CASAS GÓMEZ, M., DÍAZ HORMIGO, M. T. \& CRESPO MIGUEL, M. (2018): La investigación lingüística en Andalucia: XV Jornadas de Lingüistica. Cádiz, 8 y 9 de marzo de 2016, Cádiz: Servicio de Publicaciones de la Universidad de Cádiz.

CASAS GÓMEZ, M. (2018): "Las relaciones conceptuales como regla de comportamiento lingüístico en la identificación de usos terminológicos”, en M. Díaz, G. Vaamonde, A. Varela, M. C. Cabeza, J. M. Garcia-Miguel \& F. Ramallo (eds.): Actas do XIII Congreso Internacional de Lingüistica Xeral, Vigo: Universidad de Vigo, pp. 191-197.

CEBALLOS CASTRO, G., SÁNCHEZSAUS LASERNA, M. \& MARÍ SÁEZ, V. M. (2018): "Ejes temáticos y palabras clave en la comunicación para el desarrollo y el cambio social en España. Análisis de marcadores lingüisticos en revistas españolas de comunicación", Pragmalingüística, 26, pp. 12-31. https://doi.org/10.25267/Pragmalinguistica.2018.i26.01.

CORPAS PASTOR, G. (2017): "VIP: VoiceText Integrated System for Interpreters", en J. Esteves-Ferreira, J. Macan, R. Mitkov y O.-M. Stefanov (eds.): Proceedings of the 39th Conference Translating and the Computer, ASLING. Ginebra: Tradulex, pp. 7-10.

CRESPO MIGUEL, M. (2019): "Chatbots: technological opportunities for a teaching beyond the classroom", En J. J. GÁZQUEZ LINARES, M. M. MOLERO JURADO, A. B. BARRAGÁN MARTÍN, M. M. 
SIMÓN MÁRQUEZ, A. MARTOS

MARTÍNEZ, J. G. SORIANO

SÁNCHEZ, N. F. OROPESA RUIZ

(eds.) Innovación Docente e Investigación en Arte y Humanidades.

Madrid: Dykinson S. L. pp. 11891198.

CRESPO MIGUEL M. \& SÁNCHEZ-

SAUS LASERNA M. (2020): "Píldo-

ras formativas para la mejora educativa universitaria: el caso del Trabajo de Fin de Grado en el Grado de Lingüística y Lenguas Aplicadas de la Universidad de Cádiz", Education in the Knowledge Society (EKS), 21.

CRYSTAL, D. (2008): A Dictionary of Linguistics and Phonetics (6th ed.), Oxford: Blackwell.

JOHNSON, K. \& JOHNSON, H. (eds.) (1998): Encyclopedic dictionary of applied linguistics, Oxford: Blackwell Publishing.

FERREIRO, R. F. (2006): "El reto de la educación del siglo XXI: la generación N", Apertura, 6(5).

GARCÍA, L., RUIZ, M. \& DOMÍNGUEZ, D. (2007): De la educación a distancia a la educación virtual, Barcelona: Ariel.

GONZÁLEZ-MARTÍNEZ, J., FORTUÑO, M. \& ESPUNY-VIDAL, C. (2016):

"Las redes sociales y la educación superior: las actitudes de los estudiantes universitarios hacia el uso educativo de las redes sociales, de nuevo a examen", Education in the Knowledge Society, 17(2), pp. 2138.

HEMFORTH, B. (2017): "Experimental Linguistics", Oxford Bibliographies Online: Linguistics, NY: Oxford University Press.

MADNANI, N. (2007): "Getting started on natural language processing with Python. XRDS: Crossroads", The ACM Magazine for Students, 13(4), p. 5.

MARÍ SÁEZ, V. M. (2016): “Communication, development, and social change in Spain: A field between institutionalization and implosion", International Communication Gazette, 78(5), pp. 469-486.
MONTES SOLDADO, R., BLANCO, I., FERRER, E. M., ZEA, N. P., RODRÍGUEZ, P. P., BARRANCO, M. J. H., \& GÓMEZ-MORENO, P. U. (2014): "Hacia un nuevo modelo de uso de Moodle para la comunidad universitaria", Comunicación actual: redes sociales y lo $2.0 y$ 3.0 (pp. 401-412). Madrid: McGraw-Hill Interamericana de España.

NAVARRO T., JIMÉNEZ HIDALGO, S. \& FERNÁNDEZ MORALES, I. (2016): "Humanidades Digitales. Nuevos retos, nuevas formas de trabajar", DIGITAL.CSIC. Podcast. https://digital.csic.es/handle/10261/14057 8

NERBONNE, J. \& TONELLI, S. (2017): "Introduction to the Special Issue on Digital Humanities and Computational Linguistics", Italian Journal of Computational Linguistics, 2(2), pp. 7-10.

OZGUR, C., COLLIAU, T., ROGERS, G., HUGHES, Z. \& MYER-TYSON, B. (2017): "MatLab vs. Python vs. R", Journal of Data Science, 15(3), pp. 355-372.

POZA-LUJÁN, J. L., CALDUCH-LOSA, Á., ALBORS, A., CABRERA, M., TERUEL, D., REBOLLO, M. \& DÍEZ SOMAVILLA, R. (2014): "Propuesta de parámetros y caracterización de los grupos de las redes sociales orientados a la docencia universitaria: experiencia y resultados", Revista de Educación a Distancia, 44.

PRESNER, T. (2009): "Digital humanities manifesto 2.0 launched", Digital humanities, 22.

RAMÍREZ SALADO, M. \& ÁLVAREZ TORRES, V. (2018): "Métodos y técnicas de detección de unidades terminológica", en M. Díaz, G. Vaamonde, A. Varela, M. J. Cabeza, J. M. García-Miguel \& F. Ramallo (Eds.): Actas do XIII Congreso Internacional de Lingüística Xeral (pp. 744-749), Vigo: Universidad de Vigo.

RICHARDS, J. C., \& SCHMIDT, R. (2010): Longman Dictionary of 
Language Teaching and Applied Linguistics, Harlow: Pearson.

ROCKWELL, G., SINCLAIR, S. \& RADZIKOWSKA, M. (2020): “Text Analysis Portal for Research (TAPoR)", Education and Research Archive. Edmonton: University of Alberta.

SÁNCHEZ-SAUS LASERNA, M. (2019): "Redes sociales e innovación docente: un reto y una oportunidad para el aula de lingüística y de lenguas". En J. J. Gázquez Linares, M. M. Molero Jurado, A. B. Barragán Martín, M. M. Simón Márquez, A. Martos Martinez, J. G. Soriano Sánchez, N. F. Oropesa Ruiz (eds.), Innovación docente e investigación en arte $y \mathrm{hu}$ manidades, pp. 912-921. Madrid: Dykinson.

SÁNCHEZ-SAUS LASERNA M. \& CRESPO MIGUEL, M. (2018): "Social media as a teaching innovation tool for the promotion of interest and motivation in higher education", 2018 International Symposium on Computers in Education (SIIE) (pp. 1-5), Jerez: IEEE.
VARO VARO, C. (2018): "El devenir en la investigación del área de Lingüística General en la Universidad de Cádiz", M. Casas Gómez, M., M. T. Diaz Hormigo \& M. Crespo Miguel (2018): La investigación lingüistica en andalucía: XV jornadas de lingüística. Cádiz, 8 y 9 de marzo de 2016. Cádiz: Servicio de Publicaciones de la Universidad de Cádiz.

URSUA, N. (2016): "El pensar humanístico frente a las "humanidades digitales", Revista Interdisciplinaria de Filosofia y Psicología, 11(36), pp. 32-40.

VARSAVA, N. (2018): "Computational Legal Studies, Digital Humanities, and Textual Analysis". En R. Whalen (ed.), Computational Legal Studies: The promise and challenge of data-driven legal research, Cheltenham: Edward Elgar Publishing.

VOGEL, F., HAMANN, H. \& GAUER, I. (2018): "Computer-assisted legal linguistics: corpus analysis as a new tool for legal studies", Law y Social Inquiry, 43(4), pp. 13401363. 